\title{
The key technology research for the back-EMF method of BLDCM
}

\author{
Zhou $\mathrm{Yu}^{1, \mathrm{a}}$, Bin $\mathrm{Hu}^{2, \mathrm{~b}}$ and Hai Huan Zong ${ }^{3, \mathrm{c}}$ \\ 1,2,3 ZhengZhou Electric Power College, China

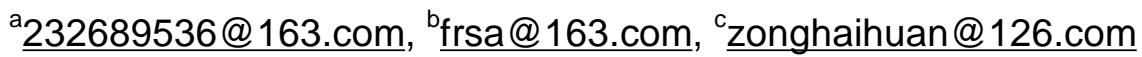

Keywords: Brushless DC motor (BLDCM); Position-sensorless; Back-EMF; zero crossing detection method.

Abstract. The direct current motor (DCM) is widely applied in industrial field. The presence of brush and commutator in traditional DCM result in sparks and noise, and may reduce working life of the motor, therefore, the research for brushless direct current motor (BLDCM) becomes hot. When the motor running, the detected electric signal is calculated to obtain the rotor position, and then achieve the purpose of accurately commutation. In this paper, the BLDCM without position sensor control is achieved with back-EMF method. Simulation results show that the method can more accurately detect the back-EMF zero crossing point, and then get the exact position signal of the rotor.

\section{Introduction}

In many applications, BLDCM requires the system is fast dynamic response, high static accuracy and strong anti-jamming capability[1]. The research of position-sensorless control is more popular of today's brushless DC motor, which can narrow the motor volume further and enhance stability, meanwhile further simplify the structure of the motor body, reducing its economic cost. With the increasing strength of research, the development and application of position-sensorless BLDCM is entering a new stage [2].

\section{Mathematical model of the BLDCM}

The position signal is obtained through the detection of physical quantities easy to be get, such as the stator voltage, current, etc., the mainly used methods are back-EMF, third harmonic potential, inductive method and state observer method. The back-EMF is currently the most mature, the most simply to achieve and the most widely used method to detect the rotor position [3], in this paper, the method is adopted to achieve the position-sensorless control of BLDCM.

As the self and mutual inductances of the stator winding are constant, the connection of three phase windings is star-shaped and no leads to the midline, thereby the constraints are as follows:

$$
i_{A}+i_{B}+i_{C}=0
$$

and:

$$
M i_{B}+M i_{C}=-M i_{A}
$$

put (1)(2) into voltage balance equation:

$$
\left\{\begin{array}{l}
u_{A}=R i_{A}+(L-M) \frac{d i_{A}}{d t}+e_{A} \\
u_{B}=R i_{B}+(L-M) \frac{d i_{B}}{d t}+e_{B} \\
u_{C}=R i_{C}+(L-M) \frac{d i_{C}}{d t}+e_{C}
\end{array}\right.
$$

According to (3), the equivalent model of BLDCM.

\section{The key technology research for the back-EMF method of BLDCM}

we can see that brushless DC motor only has two conductive switch tubes at any one time, every $60^{\circ}$, that is once commutation, when the back electromotive force of each phase reaches the maximum 
amplitude, its phase is the same as the corresponding current, and continues $120^{\circ}$ electrical angle. The $30^{\circ}$ delay of electrical angle whenever the back-EMF reach zero crossing is the next commutation moment, according to this rule, as long as the zero-crossing time of each phase back electromotive force can be detected, the commutation signal can be calculated and continued $120^{\circ}$ electrical angle. However, the brushless DC motor has no leads to the neutral point, then the size of the back electromotive force can not be directly detected, therefore, the indirect detection is needed. Based on the mathematical model of BLDCM, the equation of back electromotive force is derived as below [4].

Since the operating mode of the motor is two-two conduction with $120^{\circ}$ and only two phase windings work at any one time, so the third phase winding is disconnected. According to equation (3), the balance equation of three phase terminal voltage, each phase voltage has neutral point voltage $u_{N}$. The equation of each phase is as follow:

$$
\begin{aligned}
& u_{A}=R i_{A}+(L-M) \frac{d i_{A}}{d t}+e_{A}+u_{N} \\
& u_{B}=R i_{B}+(L-M) \frac{d i_{B}}{d t}+e_{B}+u_{N} \\
& u_{C}=R i_{C}+(L-M) \frac{d i_{C}}{d t}+e_{C}+u_{N}
\end{aligned}
$$

Assuming that phase $\mathrm{C}$ is not conducting, the current flows into phase $\mathrm{A}$ and outflows from $\mathrm{B}$, during this period, the back electromotive force of phase A is positive, phase B's is negative, the zero crossing point of phase C's back electromotive force is to be detected.

Take $\mathrm{N}$, which is the neutral point of $\mathrm{Y}$ pattern winding, as the reference potential, the phase $\mathrm{C}$ winding is out of work since the switch tube VT2 is disconnect, that means $i_{C}=0$, therefore $i_{A}=-i_{B}$, and formula (6) can simplified as follow:

$u_{C}=e_{C}+u_{N}$

When the back electromotive of phase $\mathrm{C}$ cross zero point, $e_{A}=-e_{B}$, voltage of the neutral point can be obtained:

$u_{N}=\frac{1}{2}\left(u_{A}+u_{B}\right)$

Put (8) into (7), then:

$e_{C}=u_{C}-\frac{1}{2}\left(u_{A}+u_{B}\right)$

The same principle may prove $e_{A}$ and $e_{B}$.

From above projections, we can obtain:

$e_{X}=\frac{3}{2} u_{X}-\frac{1}{2}\left(u_{A}+u_{B}+u_{C}\right) \quad X=A, B, C$

In fact, the conclusion is the same. From the equation of back electromotive force, we can see that the detection of the motor rotor position based on back electromotive force, is essentially by measuring the phase voltage of three phase or the terminal voltage of three phase, and deducing the zero crossing point of back electromotive force, so as to achieve the motor commutation accurately.

\section{Improvements of terminal voltage method}

The BLDCM use PWM chopper to control speed, since the motor commutation and the switch transform may cause variety inconsistencies of lead and lag, the terminal voltage will be affected by kinds of high-frequency interference signals, and the detection signal of back-EMF need to be filtered to obtain better waveform, especially in the control circuit, where the voltage of control signal is relatively small, therefore, bleeder measures are used in the circuit output by inverter to obtain suitable voltage signal, but this resulting in the phase deviation of back-EMF signal. So it is necessary to increase the bleeder filter circuit. 
According to the symmetry principle, here the back-EMF detecting circuit of terminal voltage changes to be the one of phase voltage. The circuit is pairwise conduction, the third phase is cutoff, therefore the sum of three phase current and three phase back-EMF is zero at any time.

Adding the three formulas in (3):

$u_{O}=u_{N}=\frac{u_{A}+u_{B}+u_{C}}{3}$

By calculation, the following relational expression can be obtained:

$$
\begin{aligned}
& u_{A^{\prime} O}=k\left(u_{A}-u_{O}\right)=\frac{2}{3} k\left[u_{A}-\frac{1}{2}\left(u_{B}+u_{C}\right)\right] \\
& u_{B^{\prime} O}=k\left(u_{B}-u_{O}\right)=\frac{2}{3} k\left[u_{B}-\frac{1}{2}\left(u_{A}+u_{C}\right)\right] \\
& u_{C^{\prime} O}=k\left(u_{C}-u_{O}\right)=\frac{2}{3} k\left[u_{C}-\frac{1}{2}\left(u_{A}+u_{B}\right)\right]
\end{aligned}
$$

According to the above conclusions, the moment that $u_{A^{\prime} O}, u_{B^{\prime} O}, u_{C^{\prime} O}$ equal to zero is just the zero crossing time of the back-EMF, and the commutation point of the BLDCM can be obtained by delaying the electrical of $30^{\circ}$, its schematic diagram is shown in Figure 1, the corresponding detailed switching order and switching process is shown in Table 1, assuming that the A-phase and B-phase are conduction at the beginning status[5].

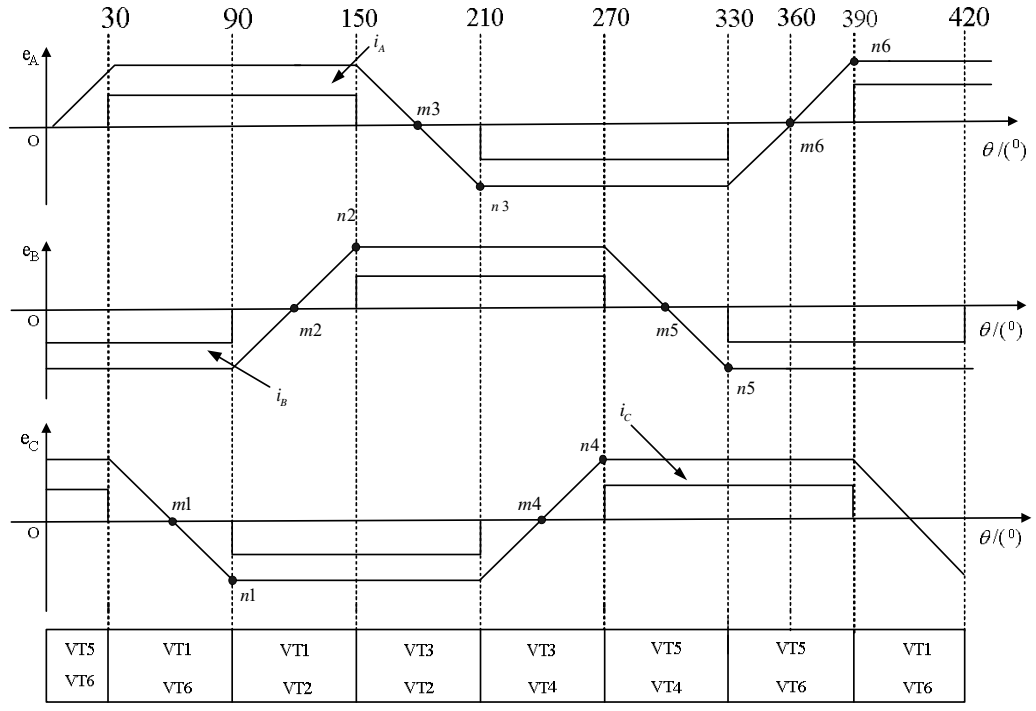

Figure 1. the schematic of commutation with $30^{\circ}$ delay

Table1. the order of the switch with $30^{\circ}$ delay

\begin{tabular}{ccccc}
\hline $\begin{array}{c}\text { zero } \\
\text { crossing } \\
\text { point }\end{array}$ & $\begin{array}{c}\text { Delay } \\
\text { angle }\end{array}$ & $\begin{array}{c}\text { commutation } \\
\text { point }\end{array}$ & $\begin{array}{c}\text { the working switching tube and the } \\
\text { Conduction phase before } \\
\text { switching phase }\end{array}$ & $\begin{array}{c}\text { the working switching tube and } \\
\text { the Conduction phase after } \\
\text { switching phase }\end{array}$ \\
\hline $\mathrm{m} 1$ & 30 & $\mathrm{n} 1$ & VT1 VT6 (A B) & VT1 VT2 (A C) \\
$\mathrm{m} 2$ & 30 & $\mathrm{n} 2$ & VT1 VT2 (A C) & VT2 VT3 (B C) \\
$\mathrm{m} 3$ & 30 & $\mathrm{n} 3$ & VT2 VT3 (B C) & VT3 VT4 (B A) \\
m4 & 30 & $\mathrm{n} 4$ & VT3 VT4 (B A) & VT4 VT5 (C A) \\
m5 & 30 & $\mathrm{n} 5$ & VT4 VT5 (C A) & VT5 VT6 (C B) \\
m6 & 30 & $\mathrm{n} 6$ & VT5 VT6 (C B) & VT6 VT1 (A B) \\
\hline
\end{tabular}

\section{Simulation results and analysis}

Set the supply voltage of the motor is $220 \mathrm{v}$, the speed is $3000 \mathrm{rpm}$, suddenly apply load $3 \mathrm{~N}^{*} \mathrm{~m} 0.2 \mathrm{~s}$ after the motor starts, the starting current of a-phase is very large, but after a very short period of shocking it is stabilized and its magnitude is relatively small, when load $3 \mathrm{~N}^{*} \mathrm{~m}$ is added at $\mathrm{t}=0.2 \mathrm{~s}$, the stator current becomes larger, as shown in Figure 2: 


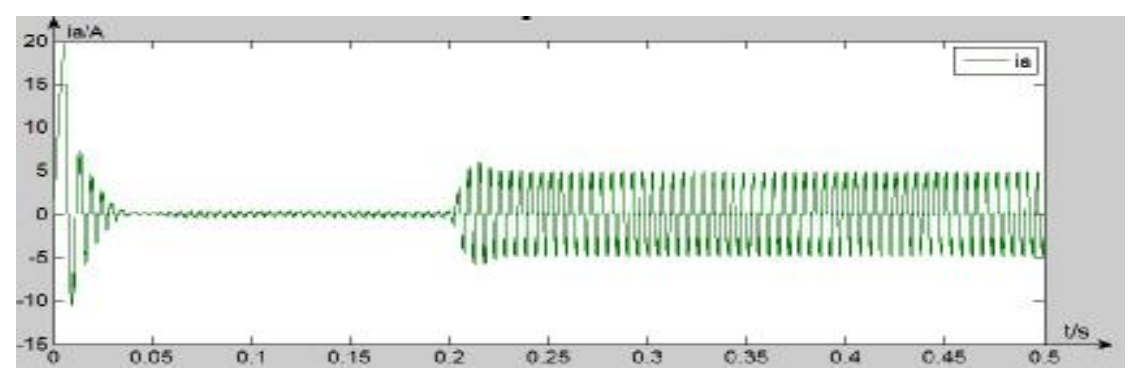

Figure2. the a-phase current of the stator

If the load mutates, the torque will increase as the current increasing, thereby the rapid adjustment of the torque is achieved, the waveform of the torque is shown as Figure 3:

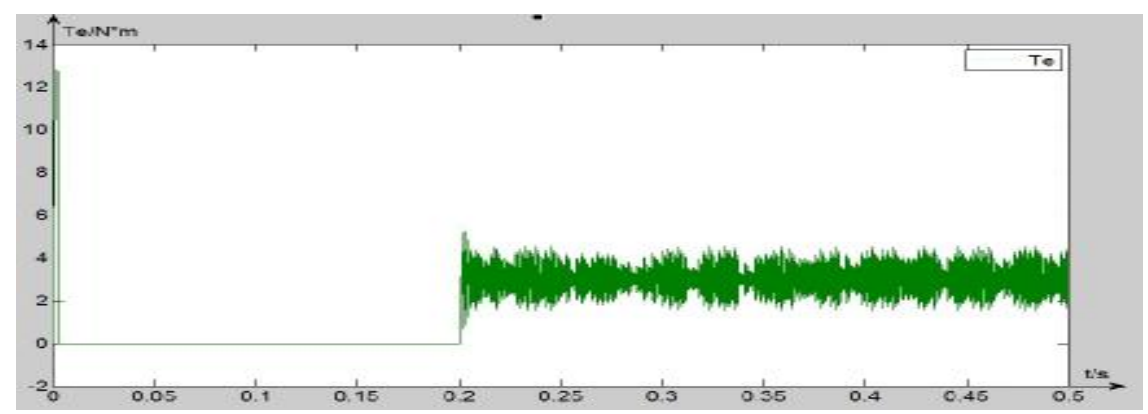

Figure 3.the waveform of the torque

Conclusion: the simulation in this paper shows that the waveform is consistent with theoretical analysis, if sudden load arises, the revolving speed could respond quickly and trend to be stable rapidly, moreover, the torque response can well follow the change of the current, the system can run smoothly and has good static and dynamic characteristics.

\section{Summary and outlook}

Based on the control method of back-EMF and the thought of combine the separately modeled control modules, this paper established a simulation control model of position-sensorless BLDCM, the simulation results show that the position-sensorless control system has favorable speed performance under the control of current speed dual closed-loop PI.

This paper adopts the improved detection method of terminal voltage, but it is not enough for the precise positioning of rotor in servo system and motion control system. Besides, some issues as the best commutation, the torque ripple and the control algorithms need to be improved in future study areas.

\section{References}

[1] Lan BaoHua, Liu JianCheng, A New Position Detection Method for Brushless DC Motors,Computer Engineering\&Science, 2009,31 (12) ,97-99

[2]Tan YaLi,Guo ZhiDa,Driver Design of BLDCM with Trapezoidal and Sinusoidal Compound Excitation,Electric Drive,2011,41 (6),11-15

[3] Song Fei, Zhou Bo, Wu XiaoJing, Closed Loop Control Method to Correct Position Phase for Sensorless Brushless DC Motor, Proceedings of the CSEE, 2009,29（12），52-57

[4] Damodharan P.,Vasudevan K. Indirect back-EMF zero crossing detection for sensorless BLDC motor operation. in:Proceedings of International Conference on Power Electronics and Drives Systems. Kuala Lumpur, 2005,1(11):1107-1111.

[5] Luo LongFu,Yang Yan,Fang RiJie, Primary Problems Worthy to Be Further Researched for BLDCM,Micro-motor,2002,35 (1) :15-16 